\title{
Search for muon to electron conversion at J-PARC MLF : Recent status on DeeMe
}

\author{
Yohei Nakatsugawa* \\ $K E K$ \\ E-mail: nakatsu@post.kek.jp
}

DeeMe is a new experiment searching for muon to electron conversion in the nuclear field and is proposed at J-PARC Materials and Life Science Experimental Facility (MLF). The signal electrons of $\mu-e$ conversion emitted from muonic atoms formed in the muon production target are transported to the spectrometer by the secondary beamline and identified by momentum analysis. The single event sensitivities achieved by this experiment are expected to be $2.1 \times 10^{-14}$ and $1.2 \times 10^{-13}$ with a silicon carbide and a graphite muon production target, respectively. We are preparing for the experiment. The simulation studies for beamline optics, background estimation were performed. The prototype of tracking device was tested using beams. The spectrometer magnet was brought in MLF. The SiC muon production target is under development. The recent status on DeeMe is reported.

16th International Workshop on Neutrino Factories and Future Neutrino Beam Facilities 25 -30 August, 2014

University of Glasgow, United Kingdom

\footnotetext{
* Speaker.
} 


\section{Introduction}

Charged Lepton Flavor Violation (cLFV) is forbidden in the original Standard Model (SM). Based on the neutrino oscillation, cLFV could be induced by neutrino flavor mixing in the intermediate state. However, the branching ratio of such process is proportional to $\left(\Delta m_{i j}^{2} / M_{W}^{2}\right)^{2}$, where $\Delta m_{i j}^{2}$ is the neutrino mass-squared difference and $M_{W}$ is the $W$-boson mass, and it is calculated to be at the level of $10^{-50}[1,2]$. This implies that such processes can not be observed experimentally and cLFV is still forbidden substantially in the framework of the SM extended for neutrino oscillation. Therefore, the experimental observations of cLFV processes gives clear evidence of new physics beyond the SM.

Muon to electron conversion in the nuclear field $\left(\mu^{-} N \rightarrow e^{-} N\right)$ is one of the cLFV processes, and is considered as one of the most powerful probes to search for cLFV. Many theoretical models, such as SUSY-GUT[3, 4], SUSY-seesaw[5], Doubly Charged Higgs[6], predict sizable branching ratios of $\mu-e$ conversion. For example, supersymmetric grand unified theory (SUSY-GUT) predicts the brancing ratio of the order of $10^{-14} \sim 10^{-18}$ based on slepton mixing.

The current upper limits for $\mu-e$ conversion are given by the experiments at TRIUMF and PSI. The upper limit for gold $\left(\mu^{-} \mathrm{Au} \rightarrow e^{-} \mathrm{Au}\right)$ is $7 \times 10^{-13}$ from the results of SINDRUM-II experiment at PSI [7], and those for titanium $\left(\mu^{-} \mathrm{Ti} \rightarrow e^{-} \mathrm{Ti}\right)$ are $4.3 \times 10^{-12}$ and $4.6 \times 10^{-12}$ from the results of SINDRUM-II [8] and an experiment at TRIUMF [9], respectively. These experimental results imply that we can reach the level of theoretical predictions by improving the experimental sensitivity by only a few orders of magnitude and the discovery is right around the corner. For these reasons, more sensitive experiments are desired to be started in a timely manner.

\section{DeeMe Experiment}

An experiment searching for $\mu-e$ conversion in the nuclear field, DeeMe, is proposed at $\mathrm{J}$ PARC Materials and Life Science Experimental Facility (MLF)[10, 11]. DeeMe experiment will be carried out using a new beamline (H-line) which is now under construction at J-PARC MLF Muon Science Establishment (MUSE). A conceptual view of DeeMe experiment is shown in Figure 2. The primary proton beam is accelerated to $3 \mathrm{GeV}$ by Rapid Cycling Synchrotron (RCS) and injected into the muon production target. The power of the beam is $300 \mathrm{~kW}$, and will be upgraded to $1 \mathrm{MW}$. Low energy muons stop in the production target and form muonic atoms. These muonic atoms are utilized to search for $\mu-e$ conversion. The signal electrons from $\mu-e$ conversion in the muonic atoms emitted in the direction of $\mathrm{H}$-line are transported to the spectrometer by the beamline. Because the signal electrons have unique momentum $(105 \mathrm{MeV} / \mathrm{c})$, they can be identified by the momentum analysis using the spectrometer. The beam optics is optimized for the momentum of the signal electrons in order to maximize the transmission efficiency of the signal electrons. At the same time, low momentum backgrounds are swept out during transportation.

A number of particles reach the spectrometer at the moment of proton irradiation on the target and these are called prompt burst. The signal electrons of $\mu-e$ conversion, on the other hand, are delayed by $\mu \mathrm{sec}$ order. The time window for analysis is then delayed by $300 \mathrm{nsec}$ from the timing of the propmt burst. Thanks to the bunched structure of the proton beam, the prompt burst can be 
rejected by selecting the delayed electrons. The beam structure is shown in Figure 2 together with the analysis time window.

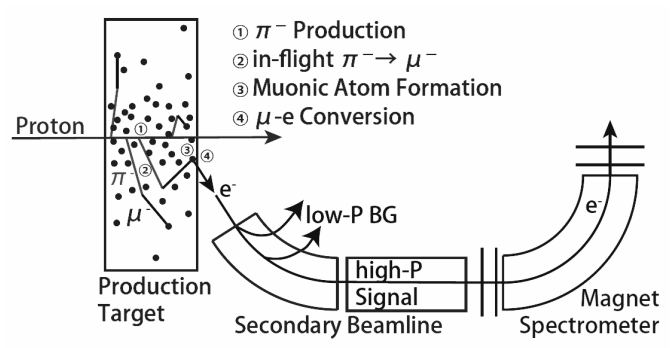

Figure 1: A conceptual view of DeeMe experiment.

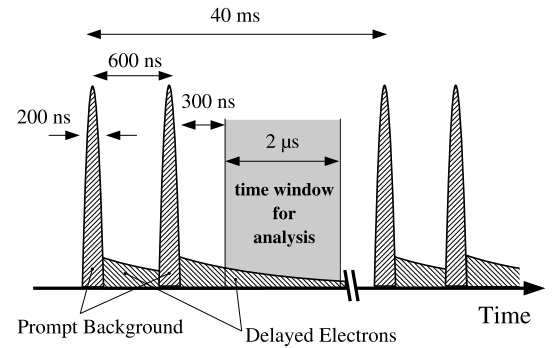

Figure 2: The bunched structure of the primary proton beam and the time window for analysis.

The possible backgrouds are Decay In Orbit, radiative pion capture, after proton induced backgrounds and cosmic ray induced backgrounds. Decay In Orbit (DIO) is Michel decay of $\mu^{-}$in $1 S$ orbit of muonic atom. Due to the recoil effect of the nucleus, the end point of DIO spectrum is extended to the momentum of the electron from $\mu-e$ conversion. The electrons can be created by radiative pion capture via the following reaction: $\pi^{-}+(A, Z) \rightarrow(A, Z-1)^{*} \rightarrow(A, Z-1)+\gamma, \gamma \rightarrow$ $e^{+} e^{-}$, and these can be rejected by selecting delayed electrons because this process take place at the prompt timing. After proton induced background is described in Sec. 6 .

\section{Beamline \& Spectrometer}

H-line is now under construnction in J-PARC MLF MUSE[12]. It is a multipurpose beamline and some experiments related to fundamental physics of muon are proposed. Figure 3 shows a schematic view of $\mathrm{H}$-line and spectrometer. The spectrometer is placed at the downstream of the beamline to measure the momentum of transported electrons. It consists of a dipole magnet (PACMAN) and four trackers. A Monte Carlo study of the beam optics was done using G4BEAMLINE package[13]. The beam optics was optimized for the momentum of the signal electrons from $\mu-e$ conversion $(105 \mathrm{MeV} / \mathrm{c})$. Figure 3 shows the acceptance of $\mathrm{H}$-line at the spectrometer as a function of momentum. The acceptance reaches more than $120 \mathrm{mSr} /(\mathrm{MeV} / \mathrm{c})$ at the signal region and the acceptable momentum region is widely spread from $90 \mathrm{MeV} / \mathrm{c}$ to $120 \mathrm{MeV} / \mathrm{c}$. Thanks to This moderate $\Delta p$, backgounds can be monitored simultaneously at higher and lower momentum regions. The former is due to after protons as explained in Sec. 6. The latter is generated by Decay In Orbit electrons, and for background estimation in the signal region, it is critically important to measure its momentum spectrum

HS1 and HB1, were installed in 2012. The PACMAN magnet which was used for PIENU experiment at TRIUMF, was transported to J-PARC and brought in MLF in August 2014. Figure 5(a) and (b) show the frontend magnets and PACMAN, respectively.

\section{Tracker}

The tracking detector is micro-cell multi-wire propotional chamber (MWPC). Tracks of electrons are reconstructed by four MWPC's for momentum analysis. In the experiment, the MWPC's 


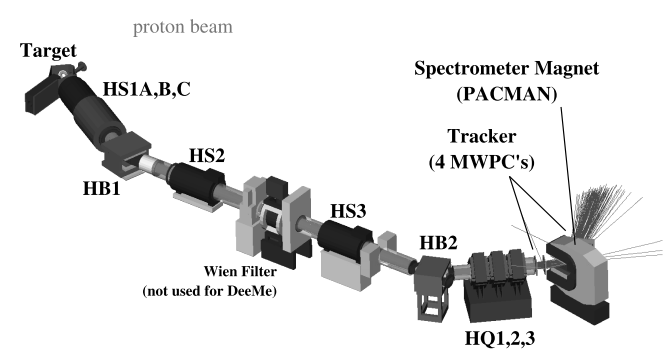

Figure 3: A schematic view of $\mathrm{H}$-line and spectrometer drawn by G4BEAMLINE. H-line consists of trasport solenoids (HS), bending dipoles (HB) and a triplet of quadrupole magnets (HQ). PACMAN is a dipole magnet. Trackers are installed at the upstream and the downstream of PACMAN.

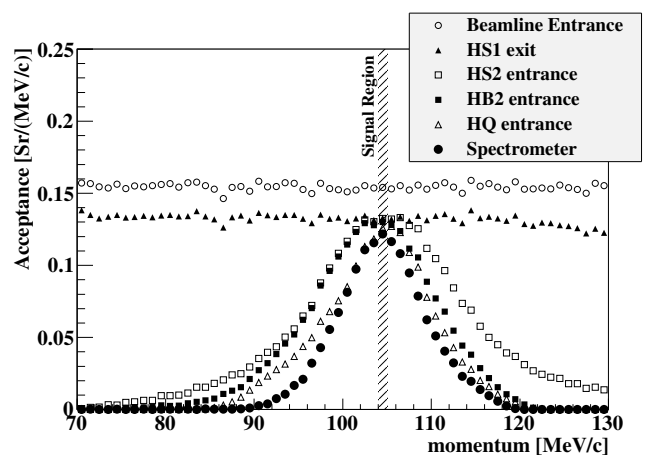

Figure 4: Acceptance of H-line at each component of the beamline as a function of the momentum. The closed circles show the acceptance for the electrons transported to the spectrometer.

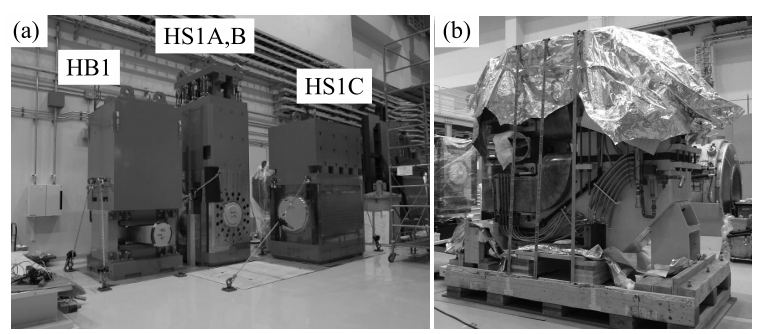

Figure 5: Pictures of magnets. (a) The frontend magnets of H-line, HS1A,B,C and HB1. (b) PACMAN magnet in MLF.

are exposed to the high rate prompt burst. The instantaneous hit rate is estimated to be an order of $10 \mathrm{GHz} / \mathrm{mm}^{2}$. The MWPC's should return to be operational in $300 \mathrm{nsec}$ after the burst to detect delayed electrons. A prototype of MWPC with an active area of $16 \mathrm{~mm} \times 16 \mathrm{~mm}$ was developed.

The beam test was done at the LINAC of Kyoto University Research Reactor Institute (KURRI). The hit rate is the same as in DeeMe experiment. The prototype was operational without any trip, but it was noticed that the gain of the MWPC is clearly reduced when the instantaneous rate of electron beam pulse within $200 \mathrm{nsec}$ is more than $100 \mathrm{MHz} / \mathrm{mm}^{2}$. This is due to space-charge effect induced by positive ions left after gas multiplication.

HV switching technique solves the space-charge problem. Avalanche multiplication induced by prompt burst is strongly suppressed by switching $\mathrm{HV}$ on the potential wires from ground to almost the same level of anode just during the burst because the electric field around anode wires becomes much weaker. A test experiment was done at J-PARC MUSE D-line in March 2014 to verify developed HV switching technique. The MWPC was irradiated with surface muon beam and the signals from anode wires were read out. In addition, a scintillation counter was placed behind the MWPC and the signals of muons penetrating the MWPC were used as reference signal. Constant voltage was applied to anode wires of the MWPC $(1100 \mathrm{~V})$ and pulsed voltage controlled by start signal of accelerator's extraction was applied to potential wires. The performance of the HV 
switching was checked changing time difference between muon pulse and the switching. Figure 6 shows that the gas gain is suppressed during the switching period.
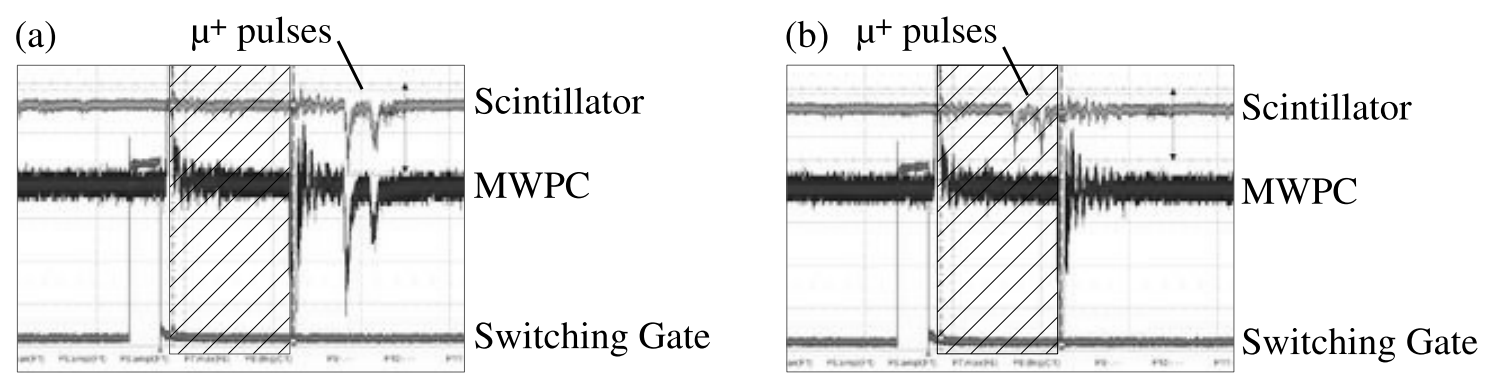

Figure 6: Waveforms of signals from the scintillation counter, the MWPC, and the switching gate. The hatched areas show HV switching periods. In Figure (a), muon pulses come after switching period and signals of the MWPC corresponding to the muon pulses can be seen. On the other hand, in Figure (b), muon pulses are covered by switching region and no signal exists in the waveform of the MWPC, while muon pulses appear in the waveform of the scintillator.

The second prototype of MWPC was fabricated. Its wire length is the same as that of the MWPC used in the physics run. Performance tests of the second prototype will be performed using muon beam of J-PARC MUSE and electron beam of KURRI LINAC. Figure 4 shows a picture of the second prototype of MWPC.

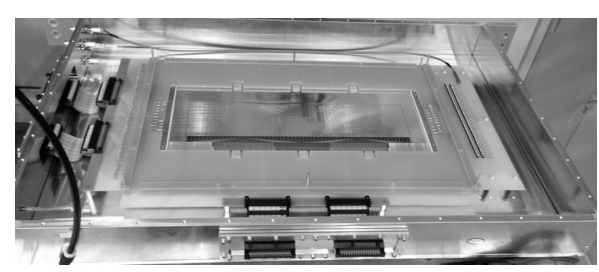

Figure 7: A picture of the second prototype of MWPC.

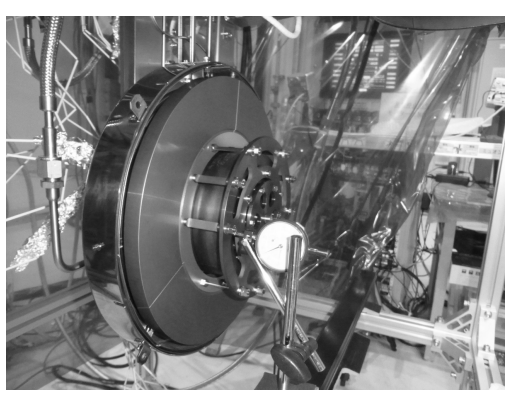

Figure 8: A picture of the prototype of $\mathrm{SiC}$ rotating target.

\section{Muon Production Target}

Currently, the muon production target made of graphite (C) is used in J-PARC MUSE. In order to maximize the physics sensitivity of the experiment, it is planned to replace the current target with a silicon carbide $(\mathrm{SiC})$ target. It is considered that $\mathrm{SiC}$ is a leading candidate material suited to the experiment because $\mathrm{SiC}$ has appropriate muonic nuclear capture rate $\left(f_{M C}\right)$ and life time of muon in a muonic atom $\left(\tau_{\mu^{-}}\right) . \mathrm{SiC}$ is used in many fields in which high performance is required, such as nuclear fusion reactor, space application, etc. The $\tau_{\mu^{-}}$is required to be longer than $300 \mathrm{nsec}$ in order to avoid the prompt burst, and the $\tau_{\mu^{-}}$of silicon and carbon is $0.76 \mu \mathrm{sec}$ and $2.03 \mu \mathrm{sec}$, 
respectively. The $f_{M C}$ of silicon is 0.62 , while that of carbon is 0.08 . Thus $\mathrm{SiC}$ gives larger muon capture rate than graphite. If the material consists of several different atoms, negative muons are divided among these atoms with the fraction of atomic muon capture $\left(f_{C}\right)$ which is propotional to the atomic number (Fermi-Teller-Z law). In case of $\mathrm{SiC}$, the fractions of muons captured by silicon and carbon nucleus are 0.7 and 0.3 , respectively, i.e. $f_{C}(\mathrm{Si})=0.7$ and $f_{C}(\mathrm{C})=0.3$. For graphite, $f_{C}(\mathrm{C})$ equal 1 because graphite is sigle element material. The number of muonic atoms formed in the material is proportional to the sum of the product of $f_{M C}$ and $f_{C}$. The $\Sigma f_{M C} \times f_{C}$ for $\mathrm{SiC}$ and graphite is 0.46 and 0.08 , respectively. This means that the experiment with a SiC target is 6 times as sensitive as that with a graphite target. The development of the $\mathrm{SiC}$ target is in progress. Figure 4 shows a picture of the prototype of $\mathrm{SiC}$ rotating target.

Because of the greater density of $\mathrm{SiC}$, the amount of heat and dose will be increased. One issue is the durability of the superconducting solenoid magnet in the other muon beamline against the increased heat. According to Monte Carlo simulation using PHITS[14], generated heat at the solenoid with the $\mathrm{SiC}$ target is estimated to be twice larger than that with the graphite target. It should be checked that the solenoid magnet is operational under the condition of use of the $\mathrm{SiC}$ taret based on data.

As the first step of DeeMe, the experiment will start with the current carbide target and the $\mathrm{SiC}$ target will be installed after accomplishment of the target and getting approval for installation from the facility.

\section{After Proton Background}

After proton stands for a proton which is extracted from RCS to MLF at the timing deviated from the beam pulse. Because the proton beam is fast-extracted, we have no after proton in principle. However, after protons may be induced by the beam halo and extracted to MLF when the extraction kicker is off. These protons could induce prompt backgrounds in the delayed timing.

After proton measurement was carried out at RCS. The beam loss monitor (BLM) is placed along side of the extraction beamline at the right downstream of RCS extraction septum magnets and detects protons outside of the extranction beamline. The BLM is composed of two scincillation counters and a Fe absorber. The Fe absorber is placed between two scintillators and the coincidence signal of those scintillators were used for counting in order to remove low energy backgrounds. Figure 9 shows a picture of the BLM.

According to a Monte Carlo study, the ratio of the number of after proton $\left(N_{A P}\right)$ to the counts of proton at BLM $\left(N_{B L M}\right)\left(f_{A P}\right)$ is estimated to be about 40 . Figure 10 shows the time distribution of the hits of BLM. The $N_{B L M}$ was obtained by integrating the histogram in the time window for analysis $(2 \mu \mathrm{sec})$. The total number of protons extracted from RCS during the measurement $\left(N_{R C S}\right)$ was counted by the accelerator group. The ratio of the number of after proton to the total number of proton extracted from RCS to MLF, $R_{A P}$, is expressed by Eq. (6.1)

$$
R_{A P}=\frac{N_{B L M} \times f}{N_{R C S}}
$$

and was measured to be $1.1 \times 10^{-18}$. As shown in Sec. 7, the after proton background was estimated using this $R_{A P}$ and its contribution was found to be very small. 


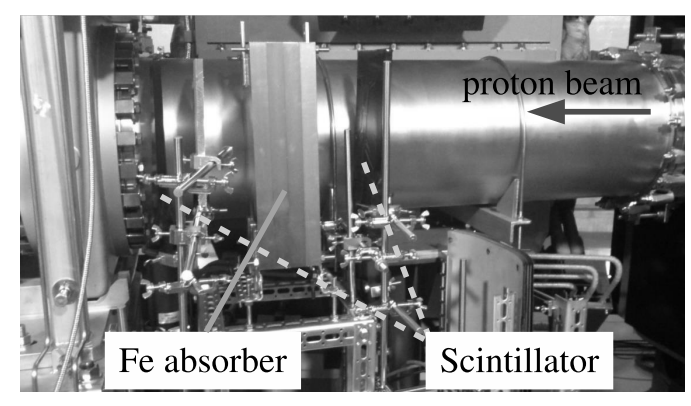

Figure 9: The setup of the beam loss monitor.

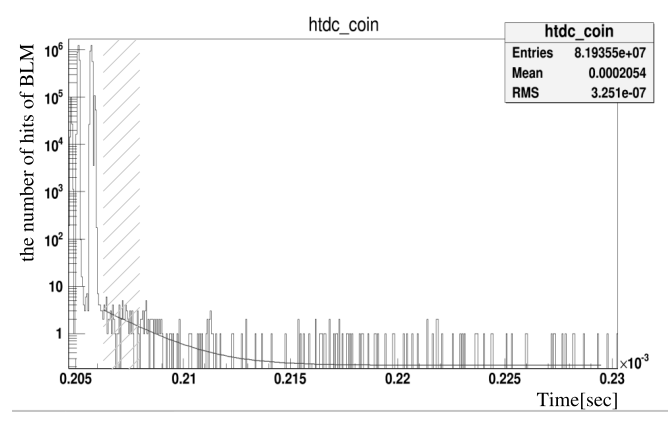

Figure 10: The time distribution of the BLM hits. The time window for analysis is indicated by the hatched area. Two peaks correspond to the normal extraction.

\section{Single Event Sensitivity}

The single event sensitivity $(S)$ is expressed by Eq. (7.1):

$$
S=\frac{1}{R_{\pi^{-}} \times f_{\pi^{-} \rightarrow \mu^{-} \text {stop }} \times f_{C} \times f_{M C} \times A_{\mu-e} \times T},
$$

where $R_{\pi^{-}}$is $\pi^{-}$a production rate per second, $f_{\pi^{-} \rightarrow \mu^{-}}$stop is a fraction of stopping muons in the production target, $f_{C}$ is the fraction of the atomic capture of muons to the atom of interest, $f_{M C}$ is the fraction of muon nuclear capture, $A_{\mu-e}$ is the total acceptance for electrons from $\mu-e$ conversion. Assuming the primary beam of $1 \mathrm{MW}$ and one year data acquisition $\left(2 \times 10^{7} \mathrm{sec}\right)$, the single event sensitivities achieved by the experiment are estimated to be $2.1 \times 10^{-14}$ and $1.2 \times 10^{-13}$ for the $\mathrm{SiC}$ and the graphite target, respectively.

The background in the signal region is evaluated based on Monte Carlo simulation to be as follows:

- Decay In Orbit: 0.09 .

- After proton: $<0.027$ (0.05 90\% C.L.), assuming $R_{A P}=10^{-18}$.

- Cosmic ray induced background: $e^{-}<0.018, \mu^{-}<0.001$.

The cosmic ray backgrounds are strongly suppressed by small live-time duty of the detector, $\frac{1}{20000}$ (primary beam of $25 \mathrm{~Hz}$, time window for analysis of $2 \mu \mathrm{sec}$ ).

The simulated momentum spectra of electron from $\mu-e$ conversion, Decay In Orbit, and after proton background for the $\mathrm{SiC}$ target are shown in Figure 11. The branching ratio of $\mu-e$ conversion is assumed to be $3 \times 10^{-14}$.

\section{Summary}

DeeMe experiment is planned at J-PARC MLF MUSE to search for $\mu-e$ conversion. A new beamline is under construction and the performance of the beamline for the experiment was 


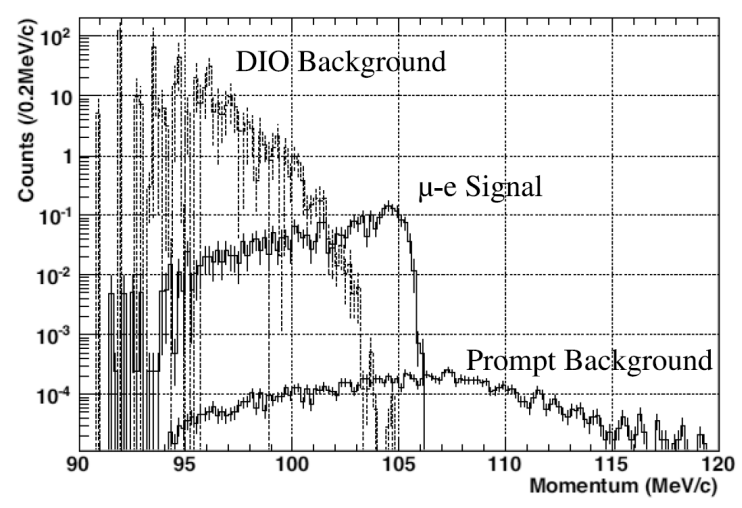

Figure 11: Momentum spectra of muon to electron conversion, Decay In Orbit, and after proton background.

evaluated based on simulation. Tracking device is developed and some performance tests were carried out. Further R\&D is ongoing. After proton background was measured and after proton ratio $\left(R_{A P}\right)$ was found to be very small. The spectrometer magnet was transported to MLF from TRIUMF. The silicon carbide target is under development. The single event sensitivities achieved by the experiment is estimated to be $2.1 \times 10^{-14}$ and $1.2 \times 10^{-13}$ for $\mathrm{SiC}$ target and graphite target, assuming $1 \mathrm{MW}$ proton beam and one year data taking. DeeMe already has Stage- 2 approval from PAC under KEK-IMSS (Institute of Materials Structure Science). The preparation of the experiment is in progress in an effort to start data taking in 2015.

\section{References}

[1] S. M. Bilenky, S. T. Petcov, and B. Pontecorvo, Phys. Lett. B 67, 309 (1977).

[2] S. T. Petcov, Yad. Fiz. 25, 641 (1977) [Sov. J. Nucl. Phys. 25, 340 (1977)].

[3] R. Barbieri and L.J. Hall, Phys. Lett. B338, 212 (1994).

[4] R. Barbieri et al., Nucl. Phys. B445, 219 (1995).

[5] K. S. Babu, Cristopher Kolda, Phys. Rev. Lett. 89, 241802 (2002).

[6] M. Raidal, A. Santamaria, Phys. Lett. B 421, 250-258 (1998).

[7] W. Burtl et al., Eur. Phys. J. C 47, 337 (2006).

[8] C. Dohmen et al., Phys. Lett. B317, 631 (1993).

[9] S. Ahmad et al., Phys. Rev. D38, 2102 (1988).

[10] M. Aoki, AIP Conference Proceedings 1441, 599 (2012).

[11] H. Natori, Nucl. Phys. B (Proc. Suppl) 284-250 (2014) 52-57.

[12] N. Kawamura et al., J. Phys.: Conf. Ser. 408 (2013) 012072.

[13] http://www.muonsinternal.com/muons3/G4beamline

[14] http://phits.jaea.go.jp/index.html. 